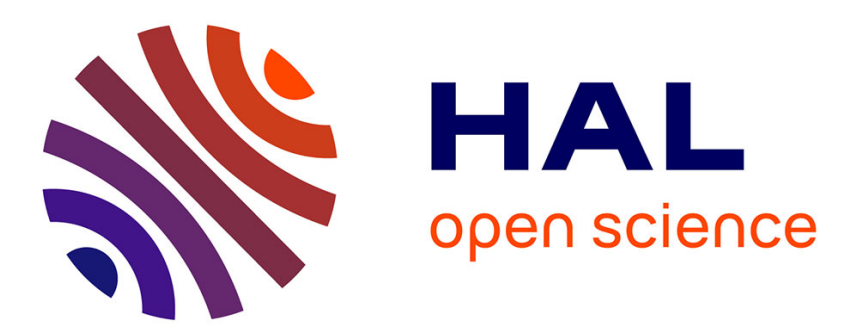

\title{
Variational Analysis in Cone Pseudo-Quasimetric Spaces and Applications to Group Dynamics
}

\author{
Truong Bao, Antoine Soubeyran
}

\section{To cite this version:}

Truong Bao, Antoine Soubeyran. Variational Analysis in Cone Pseudo-Quasimetric Spaces and Applications to Group Dynamics. Journal of Optimization Theory and Applications, 2016, 170 (2), pp.458

- 475. 10.1007/s10957-016-0933-y . hal-01690179

\section{HAL Id: hal-01690179 \\ https://hal-amu.archives-ouvertes.fr/hal-01690179}

Submitted on 1 Feb 2022

HAL is a multi-disciplinary open access archive for the deposit and dissemination of scientific research documents, whether they are published or not. The documents may come from teaching and research institutions in France or abroad, or from public or private research centers.
L'archive ouverte pluridisciplinaire HAL, est destinée au dépôt et à la diffusion de documents scientifiques de niveau recherche, publiés ou non, émanant des établissements d'enseignement et de recherche français ou étrangers, des laboratoires publics ou privés. 


\title{
Variational Analysis in Cone Pseudo-Quasimetric Spaces and Applications to Group Dynamics
}

\author{
Truong Q. Bao ${ }^{1}$ • Antoine Soubeyran ${ }^{2}$
}

\begin{abstract}
In this paper, we generalize Ekeland's variational principle in the new context of cone pseudo-quasimetric spaces. We propose this extension for applications to group dynamics in behavioral sciences. In this setting, a cone pseudo-quasimetric helps to model, in a crude way, multidimensional aspects of resistance to change for a group, where each component represents resistance to change of one agent in the group. At the behavioral level, our new version of Ekeland's variational principle shows how a group, forming and breaking routines each period by balancing between motivations and resistances to change of all members, can improve step by step their payoffs to end in a trap worthwhile to approach and reach, but not worthwhile to leave.
\end{abstract}

Keywords Multiobjective optimization - Variational principles · Variational rationality · Pseudo-quasimetric · Cone pseudo-quasimetric · Group dynamics

\section{Introduction}

The motivation of this paper is to provide a connection between Ekeland's variational principle (EVP) [1] and the existence of traps problem for group dynamics [2,3] in

Truong Q. Bao

btruong@nmu.edu

Antoine Soubeyran

antoine.soubeyran@gmail.com

1 Department of Mathematics and Computer Science, Northern Michigan University, Marquette, Michigan 49855, USA

2 CNRS and EHESS, Aix-Marseille School of Economics, Aix-Marseille University, 13002 Marseille, France 
behavioral sciences via a recent variational rationality (VR) approach of stay and change dynamics [4-6].

The pionner of group dynamic, action research and change processes is the famous psychologist Lewin $[2,3]$ who states that human behavior is a function of one person in his group environment and that "a group is composed of more than the sum of its individual members." He focuses attention on the way groups and individuals act and react to changing circumstances. His main message is the following. First, his famous balancing principle between driving and resisting forces views the dynamic of a group as a succession of quasiequilibria (statu quo), where driving forces are almost equal to resisting forces. A force can be people, resources, attitudes, traditions, regulations, values, needs, desires, etc. Second, Lewin advocates that the evolution of a group is driven by an "approach-avoidance dynamic" because there will always be driving forces that make change attractive to some people of the group and restraining forces that work to keep things as they are for other (or the same) members of the group. Then, a quasiequilibrium can change whenever driving forces are stronger than restraining forces. This view gives an informal but powerful model to explain how people and groups move through change and why they resist change. For change to happen the status quo, or equilibrium must be upset by, either strengthening the driving forces favorable to change or weakening the restraining forces. The balancing principle had a profound influence in the fields of social science, psychology, social psychology, organizational development, process management and change management. In this context, Lewin [2,3] emphasizes the major role of resistance to change using, for a typical unit change, a three stage model of change. The first stage, named "unfreezing," involves overcoming inertia, dismantling the existing "mind set," and bypassing defense mechanisms. In this initial stage, agents have to unfreeze the driving and restraining forces that hold the initial situation in a state of quasiequilibrium. The second stage is called "change." This is a transition stage of confusion where "the old ways are being challenged but we do not have a clear picture as to what we are replacing them with yet" [2]. To be able to change requires to create an imbalance between forces, i.e., to increase the drivers and to reduce the restraints. The third and final stage is "freezing." The new mindset is crystallizing, and one's comfort level is returning to previous levels. It is important to emphasize that to our knowledge the long-term Lewin's theory of group approach-avoidance dynamic has never been formalized. His initial formulation mostly uses specific examples, metaphors and no precise formalization.

The VR approach [4-6] generalizes this point of view in many different directions, providing an explicit and general model for approach-avoidance dynamics. For example driving and resisting forces refer to personal motivations and resistances to change for all agents of the group, which appear in the left-hand side and right-hand side of a worthwhile to change condition. See [7] for a well-known and short-term (but not long-term) very different formalization of approach-avoidance dynamics.

This paper, focusing explicitly the mathematical analysis on vectorial (and not scalar) worthwhile to change conditions, allows to give a crude but general model of approach-avoidance group dynamic and to give precise conditions under which the group will stop to change, reaching a variational trap, which generalizes the concept of quasiequilibrium. To achieve this aim, we develop a new version of Ekeland's 
variational principle in the setting of cone pseudo-quasimetric spaces since a cone pseudo-quasimetric can be used to measure resistance to change of a group, which is not necessarily the same for a change from $x$ to $y$ and the reverse change from $y$ to $x$ and could be zero for a real change. A challenging topological property of these spaces is that a Cauchy sequence has either no limit or more than one limit.

It is important to emphasize that a majority of generalizations of EVP have been established in a less general context of cone metric spaces, where the symmetric axiom is preserved and no more real justification is presented (see, among others, [8-12]), and that the case of cone metric spaces does not offer a true generalization of metric spaces when the cone enjoys some good properties [13-18]. The new version of EVP in cone pseudo-quasimetric established in Theorem 3.1 and its Corollary 3.1 is far-going extensions of EVP presented in [12,13] in cone metric spaces. As a consequence, the fixed-point results equivalent to Theorem 3.1 general than the corresponding results in $[11,13,16]$ in cone quasimetrics and metrics. Theorem 3.1 can be viewed as an extension of versions of EVP in [19-21] from a fixed directional pertured term $q(x, y) \xi$ to a full variable one $q_{\Theta}(x, y)$. Furthermore, the scalar version of Theorem 3.1 refines the original EVP by relaxing the lower semicontinuity assumption.

The rest of the paper is organized as follow. Section 2 presents basic definitions and preliminary results in pseudo-quasimetric spaces and cone pseudo-quasimetric spaces. In Sect. 3, we establish enhanced versions of EVP in cone pseudo-quasimetric spaces which may fail to be complete and whose limit might not be unique. The final Sect. 4 gives an application to group dynamics.

\section{Basic Definitions and Preliminaries}

\subsection{Pseudo-Quasimetric Spaces}

We use the definitions of pseudo-quasimetric spaces and the corresponding notions in these spaces such as closedness, compactness and completeness in the recent publications $[22,23]$, where the reader can find more details, discussions and references; cf. [24-27].

\section{Definition 2.1 (Pseudo-quasimetric spaces)}

(i) A bifunction $q: X \times X \rightarrow \mathbb{R}$ on a nonempty set $X$ is called a PSEUDOQUASIMETRIC, if for all $x, y, z \in X$ the following hold: (Q1) $q(x, x) \geq 0$ (nonnegativity); (Q2) $x=y \Longrightarrow q(x, y)=0$ (equality implies indistancy); and (Q3) $q(x, z) \leq q(x, y)+q(y, z)$ (triangle inequality). The pair $(X, q)$ is called a PSEUDO- QUASIMETRIC SPACE.

(ii) A pseudo-quasimetric $q$ is called a QUASIMETRIC, if it satisfies the identity of indiscernible axiom: $\left(\mathrm{Q}^{\prime}\right) x=y \Longleftrightarrow q(x, y)=0$.

(iii) A quasimetric $q$ is called a METRIC, if it satisfies the symmetry axiom: (Q4) $q(x, y)=q(y, x)$.

It is known that every pseudo-quasimetric space $(X, q)$ can be considered as a topological space in which a ball of radius $r>0$ centered at $x$ is defined by $\mathbb{B}_{q}(x, r):=\{u \in X: q(x, u)<r\}$, and the conjugate $\bar{q}$ of a pseudo-quasimetric 
$q$ defined by $\bar{q}(x, y)=q(y, x)$ for all $x, y \in X$ is also a pseudo-quasimetric. Kelly [25] called the triple $(X, q, \bar{q})$ a bitopological space. In this paper and others, we try to present definitions and results in terms of $q$ only since $q(x, y)$ and $q(y, x)$ refer to inconveniences to change from $x$ to $y$ and from $y$ to $x$ in behavioral applications.

Due to the lack of the symmetry axiom in pseudo-quasimetric spaces, each concept in metric spaces has several variants, only which needed in this paper are presented.

Definition 2.2 (Basic forward-concepts in pseudo-quasimetric spaces, [22,23]) Let $\left\{x_{n}\right\}$ be a sequence in a pseudo-quasimetric $(X, q)$. We say that:

(i) the sequence $\left\{x_{n}\right\}$ is FORWARD- CONVERGENT to $x_{*}$, if $\lim _{n \rightarrow \infty} q\left(x_{n}, x_{*}\right)=0$;

(ii) the sequence $\left\{x_{n}\right\}$ is FORWARD- CAUCHY, if

$$
\forall \varepsilon>0, \exists N_{\varepsilon} \in \mathbb{N}: q\left(x_{n}, x_{n+k}\right)<\varepsilon, \forall n \geq N_{\varepsilon}, k \in \mathbb{N}
$$

(iii) the space is FORWARD- - FORWARD- COMPLETE, if every forward-Cauchy sequence is forward-convergent to some forward limit.

The reader is referred to [22,23] for backward- and biconcepts. Note that biconcepts seem to be too restrictive in pseudo-quasimetric spaces. Indeed, it was proved by Wilson in [24, Theorems I and II] that if $\left\{x_{n}\right\}$ is both forward- and backward-convergent to $x_{*}$ in a quasimetric space $(X, q)$, then $x_{*}$ is the only limit point of $\left\{x_{n}\right\}$ of any kind, and thus, $\left\{x_{n}\right\}$ is bi-Cauchy. Many examples in [26] showed that the forward and backward notions are distinct in (asymmetric) pseudo-quasimetrics.

\subsection{Cone Pseudo-Quasimetric Spaces}

Let $Z$ be a topological vector space and $\Theta$ be an ordering cone of $Z$. Assume through out the paper that $\Theta$ is a proper $(\Theta \neq \mathbf{0}$ and $\Theta \neq Z$ ), solid (int $\Theta \neq \varnothing$ ), closed $(\Theta=\mathrm{cl} \Theta)$ and convex cone $(\Theta+\Theta=\Theta)$. We do not require that the cone $\Theta$ is either pointed $(\Theta \cap(-\Theta)=\{\mathbf{0}\})$ or normal $((\mathbb{B}+\Theta) \cap(\mathbb{B}-\Theta) \subset M \mathbb{B}$, where $\mathbb{B}$ is a unit ball and $M$ is a positive number). The Pareto order $\leq_{\Theta}$ and its weak version $<_{\Theta}$ are defined by $x \leq_{\Theta} y$ iff $x \in y-\Theta$ and $x<_{\Theta} y$ iff $x \in y-$ int $\Theta$, respectively. When $\Theta$ is not pointed, $\leq \Theta$ does not enjoy the antisymmetry axiom of a partial order since there exists $\bar{\theta}$ such that $\mathbf{0} \leq{ }_{\Theta} \bar{\theta}$ and $\bar{\theta} \leq{ }_{\Theta} \mathbf{0}$.

Definition 2.3 (Cone pseudo-quasimetrics) Let $X$ be a nonempty set, $Z$ be topological vector space and $\Theta$ be an ordering cone of $Z$. Then:

(i) A vectorial function $q_{\Theta}: X \times X \rightarrow Z$ is called a CONE PSEUDO- QUASIMETRIC, if for any $x, y, z \in X$ the following conditions hold: (Q1) $\mathbf{0} \leq \leq_{\Theta} q_{\Theta}(x, y)$; $(\mathrm{Q} 2) q_{\Theta}(x, x)=\mathbf{0}$; and $(\mathrm{Q} 3) q_{\Theta}(x, y) \leq_{\Theta} q_{\Theta}(x, z)+q_{\Theta}(z, y)$. The quadruple $\left(X, Z, \Theta, q_{\Theta}\right)$ is called a CONE PSEUDO- QUASIMETRIC SPACE.

(ii) If a cone pseudo-quasimetric $q_{\Theta}$ satisfies the coincidence axiom (Q2') $x=y$ if and only if $q_{\Theta}(x, y)=\mathbf{0}$, then it is called a CONE QUASIMETRIC.

(iii) If a cone quasimetric $q_{\Theta}$ enjoys the symmetry axiom (Q4) $q_{\Theta}(x, y)=q_{\Theta}(y, x)$, then it is called a CONE METRIC. 
Note that a cone pseudo-quasimetric space $\left(X, \mathbb{R}, \mathbb{R}_{+}, q\right)$ is nothing but a (scalar) pseudo-quasimetric space $(X, q)$ defined in Definition 2.1 .

Example 2.1 (Some cone pseudo-quasimetrics) The reader is referred to [18, pp.853854] and [16] for examples on cone metric spaces and cone quasimetric spaces, respectively.

(i) Let $X=\mathbb{R}^{2}, Z=\mathbb{R}^{2}, \Theta=\mathbb{R}_{+}^{2}$, where $\mathbb{R}_{+}^{2}=\left\{\left(\theta_{1}, \theta_{2}\right) \in \mathbb{R}^{2}: \theta_{1} \geq 0, \theta_{2} \geq 0\right\}$. Define $q_{\Theta}: X \times X \rightarrow \Theta$ by $q_{\Theta}(x, y)=\left(\max \left\{y_{1}-x_{1}, 0\right\}, \max \left\{y_{2}-x_{2}, 0\right\}\right)$ with $x=\left(x_{1}, x_{2}\right)$ and $y=\left(y_{1}, y_{2}\right)$. Obviously, $\left(\mathbb{R}^{2}, \mathbb{R}^{2}, \mathbb{R}_{+}^{2}, q_{\Theta}\right)$ is a cone pseudoquasimetric space, but not a cone quasimetric since $q_{\Theta}((1,-1),(0,-1))=$ $(0,0)$.

(ii) Let $X=\mathbb{R}, Z=\mathbb{R}^{2}$ and $\Theta=\mathbb{R}_{+}^{2}$. Define $q_{\Theta}: X \times X \rightarrow \Theta$ by $q_{\Theta}(x, y)=$ $(|x-y|, \max \{y-x, 0\})$. Then, $\left(\mathbb{R}, \mathbb{R}^{2}, \mathbb{R}_{+}^{2}, q_{\Theta}\right)$ is a cone quasimetric space.

(iii) Let $X=\mathbb{R}, Z=\mathbb{R}^{2}, \Theta=\mathbb{R}_{+}^{2}$. Define $q_{\Theta}: X \times X \rightarrow \Theta$ by $q_{\Theta}(x, y)=$ $(|x-y|, \lambda|x-y|)$ with $\lambda \geq 0$. Then, $\left(\mathbb{R}, \mathbb{R}^{2}, \mathbb{R}_{+}^{2}, q_{\Theta}\right)$ is a cone metric space.

Recently, many authors have generalized notions in metric spaces to a "more general" cone metric setting such that principal results that are well known in metric spaces still hold. We adopt them to cone pseudo-quasimetric spaces.

Definition 2.4 ([16, Definition 8], cf. $[17,18])$ Let $\left(X, Z, \Theta, q_{\Theta}\right)$ be a cone pseudoquasimetric space and $\left\{x_{n}\right\}$ be a sequence in $X$. Then:

(i) the sequence $\left\{x_{n}\right\}$ is FORWARD- CONVERGENT to $x_{*}$, if

$$
\forall \mathbf{c}<_{\Theta} \mathbf{0}, \exists N_{c} \in \mathbb{N}: q_{\Theta}\left(x_{n}, x_{*}\right)<_{\Theta} \mathbf{c}, \forall n \geq N_{c}
$$

(ii) the sequence $\left\{x_{n}\right\}$ is FORWARD- CAUCHY, if

$$
\forall \mathbf{c}<_{\Theta} \mathbf{0}, \exists N_{c} \in \mathbb{N}: q_{\Theta}\left(x_{n}, x_{n+k}\right)<\Theta \mathbf{c}, \forall n \geq N_{c}, k \in \mathbb{N}
$$

(iii) a sequence is BI- CAUCHY (or CAUCHY), if

$$
\forall \mathbf{c}<_{\Theta} \mathbf{0}, \exists N_{c} \in \mathbb{N}: q_{\Theta}\left(x_{n}, x_{m}\right)<_{\Theta} \mathbf{c}, \forall n, m \geq N_{c}
$$

(iv) the space $\left(X, Z, \Theta, q_{\Theta}\right)$ is FORWARD- - FORWARD- COMPLETE, if every forwardCauchy sequence is forward-convergent to some forward limit in $X$.

In [16], Shaddad and Noorani used the adjective "left" instead of "forward."

In [17], Jankovich et al. presented a brief reviews on cone metric spaces. It is important to emphasize that they used the completeness concept of cone metric spaces in the sense that every bi-Cauchy sequence is forward-convergent to some forward limit.

It is known from $[17,18]$ that the topological cone metric space $\left(X, Z, \Theta, d_{\Theta}\right)$ is equivalent to some topological metric space $(X, d)$ provided that $Z$ is a Banach space 
and $\Theta$ is a proper, solid, pointed, closed and convex cone. Let us list several known forms for $d$ :

$$
\begin{aligned}
& d_{1}(x, y)=\inf \left\{\|u\|: u \in \Theta \text { and } d_{\Theta}(x, y)<_{\Theta} u\right\}, \\
& d_{2}(x, y)=s_{\mathbf{e}, \Theta} \circ d_{\Theta}(x, y) \text { with } s_{\Theta, \mathbf{e}}(z):=\inf \{t \in \mathbb{R} \mid z \in t \mathbf{e}-\Theta\}, \\
& d_{3}(x, y)=\left\|d_{\Theta}(x, y)\right\| \text { provided that } \Theta \text { is a normal cone. }
\end{aligned}
$$

In this paper, we strive to establish a new and efficient vector version of EVP in cone pseudo-quasimetric spaces by constructing a forward-convergent forward-Cauchy sequence whose forward limit is a desired point of EVP. Among the aforementioned equivalent metrics, $d_{2}(x, y)=s_{\mathbf{e}, \Theta} \circ d_{\Theta}(x, y)$ seems to be the best fit to our goal because of Propositions $1-4$ given just later.

Let us recall several important properties of the nonlinear scalarization function $s_{\Theta, \mathbf{e}}$ introduced by Tammer and Weidner [29].

Let $A$ be a nonempty subset of $Z$ and $\mathbf{e} \neq \mathbf{0}$ be an element of $Z$. The function $s_{A, \mathbf{e}}: Z \rightarrow \mathbb{R} \cup\{ \pm \infty\}$ defined by

$$
s_{A, \mathbf{e}}(z):=\inf \{t \in \mathbb{R} \mid z \in t \mathbf{e}-A\}
$$

is called a NONLINEAR (SEPARATING) SCALARIZATION FUNCTION (with respect to the set $A$ and the direction $\mathbf{e})$.

Lemma 2.1 ([30, Theorem 2.3.1]) Let Z be a real topological linear space, $\Theta$ be a solid, closed, convex cone in $Z$ and $\mathbf{e} \in$ int $\Theta$. Then, the following hold:

(a) $s_{\Theta, \mathbf{e}}(z+t \mathbf{e})=s_{\Theta, \mathbf{e}}(z)+t, \forall z \in Z$ and $\forall t \in \mathbb{R}$.

(b) $s_{\Theta, \mathbf{e}}(z) \leq r$ if and only if $z \in r \mathbf{e}-\Theta$.

(c) $s_{\Theta, \mathbf{e}}(z)<r$ if and only if $z \in r \mathbf{e}-$ int $\Theta$.

(d) $s_{\Theta, \mathrm{e}}$ is positive homogeneous and continuous on $Z$.

(e) $s_{\Theta, \mathbf{e}}$ is subadditive, i.e., $s_{\Theta, \mathbf{e}}\left(z_{1}+z_{2}\right) \leq s_{\Theta, \mathbf{e}}\left(z_{1}\right)+s_{\Theta, \mathbf{e}}\left(z_{2}\right)$ for all $z_{1}, z_{2} \in Z$.

(f) $s_{\Theta, \mathrm{e}}$ is strictly $\Theta$-monotone, i.e., $\left[a \leq_{\Theta} b \wedge a \neq b \Longrightarrow s_{\Theta, \mathbf{e}}(a)<s_{\Theta, \mathbf{e}}(b)\right]$.

Below are some known results in cone metric spaces which are still valid in cone pseudo-quasimetric spaces.

Proposition 2.1 (The equivalence of scalar and cone pseudo-quasimetrics)

(i) Let $\left(X, Z, \Theta, q_{\Theta}\right)$ be a cone pseudo-quasimetric space, and let $s:=s_{\Theta, \mathbf{e}}$ be defined in (1). Then, the scalarized function $q=s \circ q_{\Theta}: X \times X \rightarrow \mathbb{R}_{+}$is a pseudo-quasimetric in $X$.

(ii) Let $(X, q)$ be a pseudo-quasimetric space, let $Z$ be a real topological space, let $\Theta$ be a solid, convex, pointed cone of $Z$, and let $\mathbf{e} \in$ int $\Theta$ be a positive direction of $Z$. Then, the bifunction $q_{\Theta}: X \times X \rightarrow Z$, whose value $q_{\Theta}(x, y)$ is an arbitrary point in the set $(q(x, y) \mathbf{e}-b d \Theta)$, is a cone pseudo-quasimetric; in particular we can have, $q_{\Theta}(x, y)=q(x, y) \mathbf{e}$ for all $x, y \in X$.

Proof The proof of (i) is simple and omitted. To prove (ii), we check the validness of conditions (Q1)-(Q3) in Definition 2.1. (Q1) and (Q2) are obvious from the definition 
of $q_{\Theta}$ and the pointedness of $\Theta$. To verify (Q3) fix arbitrary $x, y, z \in Z$. By the definition of $q_{\Theta}$, there exist $\theta_{1}, \theta_{2}, \theta_{3} \in \Theta$ such that $q_{\Theta}(x, y)=q(x, y) \mathbf{e}-\theta_{1}$, $q_{\Theta}(y, z)=q(y, z) \mathbf{e}-\theta_{2}$ and $q_{\Theta}(x, z)=q(x, z) \mathbf{e}-\theta_{3}$. Taking into account the triangle inequality of the pseudo-quasimetric $q(q(x, z) \leq q(x, y)+q(y, z))$ and the convexity of the cone $\Theta(\Theta+\Theta=\Theta)$, we have

$$
q(x, z) \mathbf{e}-\theta_{3} \in q(x, y) \mathbf{e}-\theta_{1}+q(y, z) \mathbf{e}-\theta_{2}-\Theta
$$

clearly verifying that $q_{\Theta}(x, z) \leq_{\Theta} q_{\Theta}(x, y)+q_{\Theta}(y, z)$ and the validity of the triangle inequality of the cone pseudo-quasimetric.

Proposition 2.2 (Equivalence between forward-convergence concepts) Let (X, Z, $\left.\Theta, q_{\Theta}\right)$ be a cone pseudo-quasimetric space and $q=s \circ q_{\Theta}: X \times X \rightarrow \mathbb{R}$ be a scalarized pseudo-quasimetric of $q_{\Theta}$. Then, a sequence $\left\{x_{n}\right\}$ is forward-convergent to $x_{*}$ in $\left(X, Z, \Theta, q_{\Theta}\right)$ if and only if it is forward-convergent to $x_{*}$ in $(X, q)$.

Proof Assume that $\left\{x_{n}\right\}$ is forward-convergent to $x_{*}$ in $\left(X, Z, \Theta, q_{\Theta}\right)$. By definition, for each $\mathbf{c}>_{\Theta} \mathbf{0}$, there exists $N_{c} \in \mathbb{N}$ such that $q_{\Theta}\left(x_{n}, x_{*}\right)<_{\Theta} c$ for all $n \geq N_{c}$. Then, for every $\varepsilon>0$, there exists $N_{\varepsilon}=N_{\varepsilon}$ such that for every $n \geq N_{\varepsilon}$ one has $q_{\Theta}\left(x_{n}, x_{*}\right)<_{\Theta} \varepsilon \mathbf{e} \stackrel{\text { Lem. } 2.1}{\Longrightarrow} q\left(x_{n}, x_{*}\right)=s\left(q_{\Theta}\left(x_{n}, x_{*}\right)\right)<s(\varepsilon \mathbf{e})=\varepsilon$. This means that $\left\{x_{n}\right\}$ is forward-convergent to $x_{*}$ in $(X, q)$.

Assume now that $\left\{x_{n}\right\}$ is forward-convergent to $x_{*}$ in $(X, q)$. By definition, for every $\varepsilon>0$, there exists $N_{\varepsilon} \in \mathbb{N}$ such that $q\left(x_{n}, x_{*}\right)<\varepsilon$ for all $n \geq N_{\varepsilon}$. Taking into account the properties of $s$, we get from the last inequality that

$$
q_{\Theta}\left(x_{m}, x_{m+n}\right) \in \varepsilon \mathbf{e}-\text { int } \Theta .
$$

For each $\mathbf{c}>_{\Theta} \mathbf{0} \Longleftrightarrow \mathbf{0} \in \mathbf{c}-$ int $\Theta$, since the latter set is open, we could find some $\varepsilon>0$ such that $\varepsilon \mathbf{e} \in \mathbf{c}-$ int $\Theta$. By chosing $N_{c}=N_{\varepsilon}$, one has

$$
q_{\Theta}\left(x_{m}, x_{m+n}\right) \in \varepsilon \mathbf{e}-\text { int } \Theta \subset \mathbf{c}-\text { int } \Theta,
$$

i.e., $q_{\Theta}\left(x_{m}, x_{m+n}\right)<\Theta$ c for all $n \geq N_{c}$ clearly justifying that $\left\{x_{n}\right\}$ is forwardconvergent to $x_{*}$ in $\left(X, Z, \Theta, q_{\Theta}\right)$. The proof is complete.

Proposition 2.3 (Equivalence between forward-Cauchy sequences) Let $(X, Z$, $\left.\Theta, q_{\Theta}\right)$ be a cone pseudo-quasimetric space and $q=s \circ q_{\Theta}: X \times X \rightarrow \mathbb{R}$ be the scalarized pseudo-quasimetric of $q_{\Theta}$. Then, a sequence $\left\{x_{n}\right\}$ is forward-Cauchy in $\left(X, Z, \Theta, q_{\Theta}\right)$ if and only if it is forward-Cauchy in $(X, q)$.

Proof Proceed as in Proposition 2.2.

Proposition 2.4 (Equivalence between completeness concepts) A cone pseudoquasimetric space $\left(X, Z, \Theta, q_{\Theta}\right)$ is forward-forward-complete if and only if its scalarized pseudo-quasimetric space $(X, q)$ is forward-forward-complete, where $q=s \circ q_{\Theta}$ is the scalarized pseudo-quasimetric of $q_{\Theta}$.

Proof It is immediate from Propositions 2.2 and 2.3. 


\section{Ekeland's Variational Principle in Cone Pseudo-Quasimetrics}

This section is devoted to a new version of EVP in cone pseudo-quasimetric spaces. Our approach needs not only a scalarization function to convert a vector-valued function into an extended real-valued function but also some variational techniques used in [19-21]. First, let us briefly explain why the scalarization function itself is not enough in our setting.

Let us consider a given $f: X \rightarrow Z$ and a cone pseudo-quasimetric $q_{\Theta}: X \times X \rightarrow$ $Z$. Assume that the scalarized cost function $\varphi:=s_{\Theta, \mathbf{e}} \circ f$ and the scalarized pseudoquasimetric $q:=s_{\Theta, \mathrm{e}} \circ q_{\Theta}$ satisfy all hypothesis of the original EVP. Then, it ensures the existence of $x_{*}$ for each $x_{0} \in X$ such that

(i) $\left(s_{\Theta, \mathbf{e}} \circ f\right)\left(x_{*}\right)+\lambda\left(s_{\Theta, \mathbf{e}} \circ q_{\Theta}\right)\left(x_{0}, x_{*}\right) \leq\left(s_{\Theta, \mathbf{e}} \circ f\right)\left(x_{0}\right)$;

(ii) $\left(s_{\Theta, \mathbf{e}} \circ f\right)(x)+\lambda\left(s_{\Theta, \mathbf{e}} \circ q_{\Theta}\right)\left(x_{*}, x\right)>\left(s_{\Theta, \mathbf{e}} \circ f\right)\left(x_{*}\right), \quad \forall x \neq x_{*}$.

When $q_{\Theta}(x, y)=q(x, y)$ e for some scalar pseudo-quasimetric $q(x, y)$, we have

$$
\begin{aligned}
\left(s_{\Theta, \mathbf{e}} \circ f\right)\left(x_{*}\right)+\lambda\left(s_{\Theta, \mathbf{e}} \circ q_{\Theta}\right)\left(x_{0}, x_{*}\right) & =s_{\Theta, \mathbf{e}}\left(f\left(x_{*}\right)+\lambda q_{\Theta}\left(x_{0}, x_{*}\right)\right) \\
\text { and }\left(s_{\Theta, \mathbf{e}} \circ f\right)(x)+\lambda\left(s_{\Theta, \mathbf{e}} \circ q_{\Theta}\right)\left(x_{*}, x\right) & =s_{\Theta, \mathbf{e}}\left(f(x)+\lambda q_{\Theta}\left(x_{*}, x\right)\right) .
\end{aligned}
$$

These nice properties do not hold for general cone pseudo-quasimetrics, and thus, it is impossible to formulate (i) and (ii) in terms of $f$ and $q_{\Theta}$ only.

To formulate and justify our version of EVP, we use the so-called dynamic system of $f$ and $q_{\Theta}$ with respect to $\lambda>0$ denoted by $S: X \rightrightarrows X$ and defined by

$$
S_{f, \lambda}(x):=\left\{u \in X: f(u)+\lambda q_{\Theta}(x, u) \leq_{\Theta} f(x)\right\}, \quad \forall x \in X
$$

Definition 3.1 (Generalized-Picard sequences) We say that a sequence $\left\{x_{n}\right\}$ in $X$ is a GENERALIZED- PICARD SEQUENCE of $S_{f, \lambda}$, if $x_{n+1} \in S_{f, \lambda}\left(x_{n}\right)$ for all $n \in \mathbb{N}$. It is called STRICTLY- $\Theta$-DECREASING FORWARD- CAUCHY GENERALIZED- PICARD, if $\left\{x_{n}\right\}$ is forward-Cauchy and generalized-Picard and $\left\{f\left(x_{n}\right)\right\}$ is strictly $\Theta$-decreasing, i.e., $f\left(x_{n+1}\right)<_{\Theta} f\left(x_{n}\right)$ for all $n \in \mathbb{N}$.

Theorem 3.1 (A cone pseudo-quasimetric version of EVP) Let $\left(X, Z, \Theta, q_{\Theta}\right)$ be a cone pseudo-quasimetric space, where $\Theta$ is a closed, solid, convex cone in the topological vector space $Z$. Let $s:=s_{\Theta, \mathbf{e}}$ be the nonlinear scalarization function of the ordering cone $\Theta$ along the positive direction $\mathbf{e} \in$ int $\Theta$ defined in (1), and let $q:=s \circ q_{\Theta}$ be the scalarized pseudo-quasimetric. For $f: X \rightarrow Z$ and $\lambda>0$, let $S_{f, \lambda}: X \rightrightarrows X$ be the set-valued mapping defined in (2). For a given point $x_{0} \in X$, assume that the following condition holds:

(H1) (Boundedness) $f$ is bounded from below over the set $S_{f, \lambda}\left(x_{0}\right)$; i.e., there is some element $\underline{z} \in Z$ such that $\underline{z} \leq_{\Theta} f(x)$ for all $x \in S_{f, \lambda}\left(x_{0}\right)$;

(H2) (Nonempty intersection) for any strictly $\Theta$-decreasing forward-Cauchy generalized-Picard sequence $\left\{x_{n}\right\}_{n=0}^{\infty}$ of $S_{f, \lambda}$, there exists $y \in X$ such that $S_{f, \lambda}(y) \subset S_{f, \lambda}\left(x_{n}\right)$ for all $n \in \mathbb{N}$; 
(H3) (Scalarized convergence) for any $\Theta$-decreasing generalized-Picard sequence $\left\{x_{n}\right\}_{n=0}^{\infty}$ of $S_{f, \lambda}$, if the series $\sum_{n=1}^{\infty} q_{\Theta}\left(x_{n}, x_{n+1}\right)$ is convergent in $Z$; i.e., there is $\bar{z} \in Z$ such that $\sum_{n=1}^{\infty} q_{\Theta}\left(x_{n}, x_{n+1}\right) \leq_{\Theta} \bar{z}$; then, the scalarized distances $q\left(x_{n}, x_{n+1}\right)$ tend to zero as $n \rightarrow \infty$.

Then, there is a $\Theta$-decreasing forward-Cauchy generalized-Picard sequence $\left\{x_{n}\right\}$ starting from $x_{0}$ and converging to a forward limit $x_{*}$ such that

(i) $f\left(x_{*}\right)+\lambda q_{\Theta}\left(x_{0}, x_{*}\right) \leq \Theta f\left(x_{0}\right)$ and

(ii) $f(x)+\lambda q_{\Theta}\left(x_{*}, x\right) \not \Theta_{\Theta} f\left(x_{*}\right), \quad \forall x \in X \backslash \overline{\left\{x_{*}\right\}}$,

where $\overline{\left\{x_{*}\right\}}=\left\{u \in X \mid q\left(x_{*}, u\right)=0\right\}=\left\{u \in X \mid q_{\Theta}\left(x_{*}, u\right)=\mathbf{0}\right\}$.

Furthermore, (ii) becomes (ii') $\left.f(x)+\lambda q_{\Theta}\left(x_{*}, x\right) \not\right\rfloor_{\Theta} f\left(x_{*}\right), \quad \forall x \neq x_{*}$ provided

(H4) (Limit uniqueness) for any strictly $\Theta$-decreasing forward-Cauchy generalizedPicard sequence $\left\{x_{n}\right\}_{n=0}^{\infty}$ of $S_{f, \lambda}$, it has at most one forward limit.

Proof Starting from the given element $x_{0} \in X$, we construct a forward-Cauchy generalized-Picard sequence of $S_{f, \lambda}$ which converges to some forward limit $x_{*}$ and its limit satisfies both (i) and (ii).

Given $x_{n}$. Since $x_{n} \in S_{f, \lambda}\left(x_{n}\right), S_{f, \lambda}\left(x_{n}\right) \neq \emptyset$. If $\sup _{x \in S_{f, \lambda}\left(x_{n}\right)} q\left(x_{n}, x\right)=0$, then $x_{n+1}=x_{n}$; otherwise, $x_{n+1} \in S_{f, \lambda}\left(x_{n}\right)$ such that

$$
q\left(x_{n}, x_{n+1}\right) \geq \sup _{x \in S_{f, \lambda}\left(x_{n}\right)} q\left(x_{n}, x\right)-2^{-n}, \forall n \in \mathbb{N}_{0}:=\mathbb{N} \cup\{0\}
$$

Consider two cases:

Case 1 . The sequence $\left\{x_{n}\right\}$ is eventually constant; i.e., there is $n_{*} \in \mathbb{N}$ such that $x_{n}=x_{*}$ for all $n \geq n_{*}$. In this case, $x_{*} \in S_{f, \lambda}\left(x_{0}\right), \sup _{x \in S_{f, \lambda}\left(x_{*}\right)} q\left(x_{*}, x\right)=0$ and $S_{f, \lambda}\left(x_{*}\right) \subset \overline{\left\{x_{*}\right\}}$.

Obviously, (i) and (ii) are satisfied by $x_{*}$.

Case 2. All the (scalarized) forward distances of the sequence $x_{n}$ are nonzero, i.e., $q\left(x_{n}, x_{n+1}\right)>0$ for all $n \in \mathbb{N}_{0}$. By Lemma 2.1(c), $q_{\Theta}\left(x_{n}, x_{n+1}\right) \in$ int $\Theta$. This together with $x_{n+1} \in S_{f, \lambda}\left(x_{n}\right)$ yields $f\left(x_{n+1}\right)<_{\Theta} f\left(x_{n}\right)$. Thus, $\left\{x_{n}\right\}$ is a strictly $\Theta$-decreasing generalized-Picard sequence of $S_{f, \lambda}$. It enjoys the following properties:

(a) $S_{f, \lambda}\left(x_{n+1}\right) \subset S_{f, \lambda}\left(x_{n}\right)$ for all $n \in \mathbb{N}_{0}$. It is straightforward from the definition of $S_{f, \lambda}$ and the triangle inequality of $q$.

(b) $\lim _{n \rightarrow \infty} q\left(x_{n}, x_{n+1}\right)=0$ and $\lim _{n \rightarrow \infty} \sup _{x \in S_{f, \lambda}\left(x_{n}\right)} q\left(x_{n}, x\right)=0$.

For any $n \in \mathbb{N}_{0}, x_{n+1} \in S_{f, \lambda}\left(x_{n}\right)$ yields $f\left(x_{n+1}\right)+\lambda q_{\Theta}\left(x_{n}, x_{n+1}\right) \leq \Theta f\left(x_{n}\right)$. Summing up these inequalities from $n=0$ to $m-1$ gives

$$
\lambda \sum_{n=0}^{m-1} q_{\Theta}\left(x_{n}, x_{n+1}\right) \leq_{\Theta} f\left(x_{0}\right)-f\left(x_{m}\right) \leq_{\Theta} f\left(x_{0}\right)-\underline{z},
$$

where the last estimate holds due to the boundedness condition (H1). Passing to limit as $m \rightarrow \infty$ ensures the boundedness from above of the series $\sum_{n=0}^{\infty} q_{\Theta}\left(x_{n}, x_{n+1}\right)$. 
By the convergence condition (H3), the series $\sum_{n=0}^{\infty} q\left(x_{n}, x_{n+1}\right)$ is convergent and thus $\lim _{n \rightarrow \infty} q\left(x_{n}, x_{n+1}\right)=0$ due to the divergence theorem. Then, we get from the inequality in (3) that

$$
0 \leq \lim _{n \rightarrow \infty} \sup _{x \in S_{f, \lambda}\left(x_{n}\right)} q\left(x_{n}, x\right) \leq \lim _{n \rightarrow \infty}\left(q\left(x_{n}, x_{n+1}\right)+2^{-n}\right)=0
$$

(c) $\left\{x_{n}\right\}$ is a forward-Cauchy sequence in $(X, q)$. The convergence of the series $\sum_{n=0}^{\infty} q\left(x_{n}, x_{n+1}\right)$ says that for every $\varepsilon>0$, there is $N_{\varepsilon} \in \mathbb{N}$ such that for all $m \geq N_{\varepsilon}$ and for all $k \in \mathbb{N}$ we have

$$
q\left(x_{m}, x_{m+k}\right) \leq \sum_{n=m}^{m+k-1} q\left(x_{n}, x_{n+1}\right) \leq \sum_{n=m}^{\infty} q\left(x_{n}, x_{n+1}\right)<\varepsilon,
$$

clearly verifying that $\left\{x_{n}\right\}$ is a forward-Cauchy sequence in $(X, q)$.

(d) $\left\{x_{n}\right\}$ has a forward limit which satisfies (i) and (ii).

Employing the nonempty intersection condition (H2) to the chosen strictly $\Theta$ decreasing forward-Cauchy generalized-Picard sequence $\left\{x_{n}\right\}$, we obtain the existence of $x_{*} \in X$ such that

$$
x_{*} \in S_{f, \lambda}\left(x_{*}\right) \subset S_{f, \lambda}\left(x_{n}\right), \forall n \in \mathbb{N}_{0} .
$$

Obviously, (i) holds by taking $n=0$ in (5). To justify the validity of (ii), it is sufficient to check $S_{f, \lambda}\left(x_{*}\right) \subset \overline{\left\{x_{*}\right\}}$. Indeed, for any $y_{*} \in S_{f, \lambda}\left(x_{*}\right)$, we get from (5), (a) and (b) that

$$
0 \leq q\left(x_{*}, y_{*}\right) \leq \sup _{y \in S_{f, \lambda}\left(x_{n}\right)} q\left(x_{n}, y\right) \downarrow 0 \text { as } n \rightarrow \infty
$$

Therefore, $q\left(x_{*}, y_{*}\right)=0$ and $y_{*} \in \overline{\left\{x_{*}\right\}}$. Since $y_{*}$ was arbitrary in $S_{f, \lambda}\left(x_{*}\right)$, we have $S_{f, \lambda}\left(x_{*}\right) \subset \overline{\left\{x_{*}\right\}}$.

Since (H4) implies $\overline{\left\{x_{n}\right\}}=\left\{x_{*}\right\}$, (ii) reduces to (ii'). The proof is complete.

Proposition 3.1 (Sufficient conditions for (H3)) The fulfillment of one of the following conditions

$\left(\mathbf{H 3}^{\prime}\right) \mathbf{0} \notin \operatorname{cl} \operatorname{conv}(\Theta \backslash[\mathbf{0}, \mathbf{e}))$, where $[\mathbf{0}, \mathbf{e}):=(\mathbf{0}+\Theta) \cap(\mathbf{e}-$ int $\Theta)$;

$\left(\mathbf{H 3}^{\prime \prime}\right) \exists z^{*} \in Z^{*}, \exists \gamma>0: z^{*}(z) \geq \gamma, \forall z \in \Theta \backslash[\mathbf{0}, \mathbf{e})$

implies the validity of the convergence condition (H3) in Theorem 3.1.

Proof Since $\left(\mathrm{H}^{\prime}\right) \Longrightarrow\left(\mathrm{H}^{\prime \prime}\right)$ thanks to classical separation theorems, it remains to justify $\left(\mathrm{H}^{\prime \prime}\right) \Longrightarrow(\mathrm{H} 3)$.

Arguing by contradiction, assume that (H3 $\left.{ }^{\prime \prime}\right)$ holds, but (H3) does not. Then, there are $\bar{z} \in Z$ such that $\sum_{n=1}^{\infty} q_{\Theta}\left(x_{n}, x_{n+1}\right) \leq_{\Theta} \bar{z}$, but the quasidistances $q\left(x_{n}, x_{n+1}\right)$ 
does not converge to zero; i.e., there are a positive number $\varepsilon$ and an integer number $N_{\varepsilon}$ such that $q\left(x_{n}, x_{n+1}\right)=s\left(q_{\Theta}\left(x_{n}, x_{n+1}\right)\right)>\varepsilon, \forall n \geq N_{\varepsilon}$.

By Lemma 2.1, for any $n \geq N_{\varepsilon}$ we have

$$
q_{\Theta}\left(x_{n}, x_{n+1}\right) \notin \varepsilon \mathbf{e}-\text { int } \Theta \Longrightarrow \frac{1}{\varepsilon} q_{\Theta}\left(x_{n}, x_{n+1}\right) \in \Theta \backslash[\mathbf{0}, \mathbf{e})
$$

This together with the imposed condition $\left(\mathrm{H}^{\prime \prime}\right)$ implies

$$
\frac{1}{\varepsilon} z^{*}\left(\sum_{n=N_{\varepsilon}}^{m} q_{\Theta}\left(x_{n}, x_{n+1}\right)\right)=\sum_{n=N_{\varepsilon}}^{m} z^{*}\left(\frac{1}{\varepsilon} q_{\Theta}\left(x_{n}, x_{n+1}\right)\right) \geq\left(m-N_{\delta}\right) \gamma
$$

for all $m \geq N_{\varepsilon}$. Passing to limit as $m \rightarrow \infty$, we arrive at

$$
z^{*}\left(\sum_{n=0}^{\infty} q_{\Theta}\left(x_{n}, x_{n+1}\right)\right) \geq z^{*}\left(\sum_{n=N_{\varepsilon}}^{\infty} q_{\Theta}\left(x_{n}, x_{n+1}\right)\right)=\infty
$$

contradicting the boundedness of the series $z^{*}\left(\sum_{n=1}^{\infty} q_{\Theta}\left(x_{n}, x_{n+1}\right)\right) \leq z^{*}(\bar{z})<\infty$. This contradiction proves the validity of (H3). The proof is complete.

Next, we provide an example illustrating that the condition (H3) is satisfied by closed, solid and convex cones being not pointed (and thus not normal).

Example 3.1 Let $Z=\mathbb{R}^{2}$ and $\Theta=\mathbb{R}_{+} \times \mathbb{R}$. The cone $\Theta$ is not pointed since $\Theta \cap(-\Theta)=\{0\} \times \mathbb{R}$. By chosing $\mathbf{e}=(1,0) \in$ int $\Theta$, we have

$$
(0,0) \notin \operatorname{cl} \operatorname{conv}(\Theta \backslash[\mathbf{0}, \mathbf{e}))=[1,+\infty) \times(-\infty,+\infty)
$$

and thus the validity of $\left(\mathrm{H}^{\prime}\right)$.

Take $z^{*}=(1,0)$. For any $z=\left(z_{1}, z_{2}\right) \in \operatorname{cl} \operatorname{conv}(\Theta \backslash[\mathbf{0}, \mathbf{e}))=[1,+\infty) \times(-\infty,+\infty)$, $z_{1} \geq 1, z_{2} \in \mathbb{R}$ and $z^{*}(z)=z_{1} \geq 1$ justifying the validity of $\left(\mathrm{H} 3^{\prime \prime}\right)$.

The next proposition provides a cone pseudo-quasimetrics which unconditionally satisfy the convergence condition (H3).

Proposition 3.2 Let $q$ be a pseudo-quasimetric on a nonempty set $X$, and $\Theta$ be a convex ordering cone (which is not necessarily solid) of a topological vector space $Z$. Then, the cone pseudo-quasimetric defined by $q_{\Theta}(x, y):=q(x, y) \mathbf{e}$ for some element $\mathbf{e} \in \Theta$ satisfies the scalarized convergence condition $(\mathrm{H} 3)$.

Proof It is immediate from the structure of the metric.

Next, we provide a sufficient condition for $(\mathrm{H} 2)$ in terms of lower semicontinuity.

Definition 3.2 (Strictly $\Theta$-decreasing forward-lower semicontinuity) Let ( $X, Z$, $\left.\Theta, q_{\Theta}\right)$ be a cone pseudo-quasimetric space. The function $f: X \rightarrow Z$ is said to 
be STRICTLY $\Theta$-DECREASING FORWARD- LOWER SEMICONTINUOUS on $X$ if, for any sequence $\left\{x_{n}\right\}$ in $X$ such that it is forward-convergent to $x_{*}$ and its image sequence $\left\{f\left(x_{n}\right)\right\}$ is strictly $\Theta$-decreasing, the validity of $f\left(x_{n}\right) \leq \Theta$ c for some $\mathbf{c} \in Z$ and for all $n \in \mathbb{N}$ implies $f\left(x_{*}\right) \leq{ }_{\Theta}$ c.

Proposition 3.3 (A sufficient condition for $(\mathrm{H} 2)$ ) Let $\left(X, Z, \Theta, q_{\Theta}\right)$ be a cone pseudoquasimetric space and $q=s \circ q_{\Theta}$ be the scalarized pseudo-quasimetric of $q_{\Theta}$. Assume that $(X, q)$ is forward-forward-complete and that the vectorial function $f: X \rightarrow Z$ is strictly $\Theta$-decreasing forward-lower-semicontinuous on $S_{f, \lambda}\left(x_{0}\right)$, where $S_{f, \lambda}$ is defined by (2). Then, the condition (H2) in Theorem 3.1 holds.

Proof Fix an arbitrary strictly $\Theta$-decreasing forward-Cauchy generalized-Picard sequence $\left\{x_{n}\right\}_{n=0}^{\infty}$ of $S_{f, \lambda}$. Since the space $(X, q)$ is complete by Proposition 2.4, there exists $x_{*} \in X$ such that $q\left(x_{n}, x_{*}\right) \rightarrow 0$ as $n \rightarrow \infty$. Since $\left\{x_{n}\right\}$ is a generalizedPicard sequence of $S_{f, \lambda}$, we have $x_{n+1} \in S\left(x_{n}\right)$ for all $n \in \mathbb{N}_{0}$. By the definition of $S_{f, \lambda}$, we have

$$
f\left(x_{n+1}\right)+\lambda q_{\Theta}\left(x_{n}, x_{n+1}\right) \leq_{\Theta} f\left(x_{n}\right), \forall n \in \mathbb{N}_{0} .
$$

Summing up these inequalities and using the triangle inequality of $q_{\Theta}$, we have

$$
f\left(x_{m+k+l}\right)+\lambda q_{\Theta}\left(x_{m}, x_{m+k}\right) \leq_{\Theta} f\left(x_{m+k+l}\right)+\lambda \sum_{n=m}^{m+k+l-1} q_{\Theta}\left(x_{n}, x_{n+1}\right) \leq_{\Theta} f\left(x_{m}\right)
$$

for all $m, k, l \in \mathbb{N}$, which leads to

$$
f\left(x_{m+k+l}\right) \leq_{\Theta} f\left(x_{m}\right)-\lambda q_{\Theta}\left(x_{m}, x_{m+k}\right), \forall l \in \mathbb{N} .
$$

By the assumed strict- $\Theta$-decreasing forward-lower semicontinuity of $f$, we have

$$
f\left(x_{*}\right) \leq_{\Theta} f\left(x_{m}\right)-\lambda q_{\Theta}\left(x_{m}, x_{m+k}\right) .
$$

By the triangle inequality of $q_{\Theta}$, we get from the last inequality that

$$
f\left(x_{*}\right)+\lambda\left(q_{\Theta}\left(x_{m}, x_{*}\right)-q\left(x_{m+k}, x_{*}\right)\right) \leq_{\Theta} f\left(x_{*}\right)+\lambda q_{\Theta}\left(x_{m}, x_{m+k}\right) \leq_{\Theta} f\left(x_{m}\right) .
$$

Since $k$ was arbitrary and $\Theta$ is a closed set, passing to limit we get

$$
f\left(x_{*}\right)+\lambda q_{\Theta}\left(x_{m}, x_{*}\right) \leq \Theta f\left(x_{*}\right),
$$

i.e., $x_{*} \in S_{f, \lambda}\left(x_{m}\right)$. Since $m$ was arbitrary, (H2) holds. The proof is complete.

It is easy for us to formulate a simple vectorial version of EVP in cone pseudo-quasimetric spaces $\left(X, Z, \Theta, q_{\Theta}\right)$ which reduces to the original one when $\left(X, Z, \Theta, q_{\Theta}\right)=\left(X, \mathbb{R}, \mathbb{R}_{+},|\cdot|\right)$. 
Corollary 3.1 (An enhanced version of EVP for cone pseudo-quasimetrics) Let $\left(X, Z, \Theta, q_{\Theta}\right), f, \Theta, \mathbf{e}, s=s_{\Theta, \mathbf{e}}, q=s \circ q_{\Theta}$ and $S_{f, \lambda}$ as in Theorem 3.1. Given $x_{0} \in X$ and $\lambda>0$. Assume that the following conditions hold:

(H1) $f$ is $\Theta$-bounded from below over $S_{f, \lambda}\left(x_{0}\right)$;

$\left(\mathbf{H 2}^{\prime}\right)\left(X, Z, \Theta, q_{\Theta}\right)$ is forward-forward-complete and $f$ is strictly $\Theta$-decreasing forward-lower-semicontinuous on $S_{f, \lambda}\left(x_{0}\right)$;

$\left(\mathbf{H 3}^{\prime}\right) \mathbf{0} \notin \operatorname{cl} \operatorname{conv}(\Theta \backslash[\mathbf{0}, \mathbf{e}))$ with $[\mathbf{0}, \mathbf{e})=(\mathbf{0}+\Theta) \cap(\mathbf{e}-\operatorname{int} \Theta)$.

Then, there is $x_{*} \in X$ such that

(i) $f\left(x_{*}\right)+\lambda q_{\Theta}\left(x_{0}, x_{*}\right) \leq_{\Theta} f\left(x_{0}\right)$ and

(ii) $f(x)+\lambda q_{\Theta}\left(x_{*}, x\right) \not \leq \Theta f\left(x_{*}\right), \quad \forall x \notin \overline{\left\{x_{*}\right\}}$,

where $\overline{\left\{x_{*}\right\}}=\left\{u \in X: q\left(x_{*}, u\right)=0\right\}$.

Proof It is immediate from Theorem 3.1, and Propositions 3.1 and 3.3.

Corollary 3.2 (A forward version of EVP) Let $(X, q)$ be a pseudo-quasimetric space and let $\varphi: X \rightarrow \mathbb{R} \cup\{+\infty\}$ be a proper extended real-valued function. Assume that the space $(X, q)$ is strictly $\varphi$-decreasing forward-complete, the function $\varphi$ is proper, bounded from below, and strictly decreasing forward-lower-semicontinuous. Given $x_{0} \in \operatorname{dom} \varphi$ and $\lambda>0$, consider a set-valued mapping $S_{\varphi, \lambda}: X \rightrightarrows X$ defined by (2) with $f=\varphi$ and $\leq_{\Theta}=\leq$. Then, there is $x_{*} \in X$ such that

(i) $\varphi\left(x_{*}\right)+\lambda q\left(x_{0}, x_{*}\right) \leq \varphi\left(x_{0}\right)$;

(ii) $\varphi(x)+\lambda q\left(x_{*}, x\right)>\varphi\left(x_{*}\right), \quad \forall x \in X \backslash \overline{\left\{x_{*}\right\}}$,

where $\overline{\left\{y_{*}\right\}}=\left\{u \in X: q\left(y_{*}, u\right)=0\right\}$.

Proof It is straightforward from Corollary 3.1 in the space $\left(X, \mathbb{R}_{,} \mathbb{R}_{+}, q\right), \Theta=\mathbb{R}_{+}$, $\mathbf{e}=1$, and $s_{e, \Theta}(t)=t$ for all $t \in \mathbb{R}$.

Let us conclude this section and the paper with some comparisons and remarks.

Remark 3.1 (a) To the best of our knowledge, Theorem 3.1 and Corollary 3.1 are new in two regards: (1) the results are established for cone pseudo-quasimetric spaces in which a forward-convergent sequence may have two distinct forward limits; (2) the cost vector-valued function enjoys the strictly $\Theta$-decreasing forward-lower semicontinuity property which is less restrictive than all the existing kinds of lower semicontinuity. Corollary 3.2 is not as strong as [23, Corollary 3.14] and [28, Theorem 2(3)]. They were characterizations for the completeness of pseudo-quasimetric spaces and quasimetric spaces, respectively.

(b) Although cost functions and cone pseudo-quasimetrics are single-valued, the obtained results can be extended to set-valued mappings and set-valued cone pseudoquasimetrics, respectively. Recall from [21, Definition 5.1] that a mapping $D: X \times$ $X \rightrightarrows Z$ is said to be a set-valued $\Theta$-quasimetric, if

(D1) $D\left(x_{1}, x_{2}\right) \subset \Theta$ for all $x_{1}, x_{2} \in X$;

(D2) $\mathbf{0} \in D(x, x)$ for all $x \in X$;

(D3) $D\left(x_{1}, x_{2}\right)+D\left(x_{2}, x_{3}\right) \subset D\left(x_{1}, x_{3}\right)+\Theta$ for all $x_{1}, x_{2}, x_{3} \in X$. 
(c) The convergence condition (H3) has its root in [31], where one of the first vectorial versions of EVP was established in terms of vector-valued distances. Precisely, consider an ordered space $(Z, \preceq)$ and a distance $d: X \times X \rightarrow Z$. The space $X$ is called to be $d$-complete if

$$
\sum_{n=1}^{m} q\left(x_{n}, q_{n+1}\right) \preceq \bar{z} \text { for some } \bar{z} \in Z \text {, for all } m \in \mathbb{N}
$$

implies that the sequence $\left\{x_{n}\right\}$ converges to some $x_{*} \in X$.

In this direction, Nemeth further developed them in [32] for distances $d_{\Theta}: G \times G \rightarrow$ $\Theta$ from an ordered topological Abelian group $G$ with closed positive quasiwegde $\Theta$ in $G$. A similar condition of (H3)-type is that there exists a neighborhood $U$ of the zero element such that any sequence $\left\{x_{n}\right\}$ with the property $d_{\Theta}\left(x_{n}, x_{n+1}\right) \in \Theta \backslash U$ implies that the set

$$
\left\{\sum_{n=1}^{m} d_{\Theta}\left(x_{n}, x_{n+1}\right), n \in \mathbb{N}\right\}
$$

cannot be $\Theta$-bounded from above.

(d) In contrast to our approach, the authors in [21,33-35] worked with two given spaces: a pseudo-quasimetric $(X, q)$ with $q: X \times X \rightarrow \mathbb{R}_{+}$and a cone quasimetric $D$ : $X \times X \rightarrow \Theta$ defined in (a), where $\Theta$ is an ordering cone of some normed space $Z$, and imposed the so-called convergence comparison condition; see [21, Theorem 5.2 (F3)]: for any $\Theta$-decreasing sequence $\left\{x_{n}\right\}$, the upper bound of the series

$$
\sum_{n=1}^{\infty} D\left(x_{n}, x_{n+1}\right) \subset M-\Theta,
$$

with some bounded set $M$ in $Z$ ensures that $q\left(x_{n}, x_{n+1}\right) \rightarrow 0$ as $n \rightarrow \infty$. Obviously, if $q$ happens to be the scalarized pseudo-quasimetric of $D$, then this condition is identical to the condition (H3). It is proved in [21, Theorem 5.3] that the convergence comparison condition is satisfied provided that there exist $z^{*} \in \Theta^{+}$and $\eta: \mathbb{R}_{+} \rightarrow \mathbb{R}_{+}$ such that

$$
\inf \left\{z^{*}(z): z \in \cup_{q\left(x_{1}, x_{2}\right) \geq \delta} D\left(x_{1}, x_{2}\right)\right\} \geq \eta(\delta)>0 \text { for all } \delta>0 .
$$

It seems to be more complicated than the sufficient conditions presented in Proposition 3.1.

\section{An Application to Group Dynamics}

\subsection{A Worthwhile Stay or Change Group Dynamic}

Let us define the simplest (not hierarchical) worthwhile stay or change group dynamic, using the VR concepts and modelization, see [6] for a general formulation. Consider a group of agents $j \in J \subset \mathbb{N}$ and its dynamic. In the last and current periods $n$, 
$n+1$, each agent $j$ carries out a last action $x_{n}^{j}$ and a current action $x_{n+1}^{j}$. Then, in the current period $n+1$, given their past experiences $e=e_{n}=\left(e_{n}^{j}\right)_{j \in J}$, agents moves from the profile of last actions $x=x_{n}=\left(x_{n}^{j}\right)_{j \in J}$ to carry out the current profile of actions $y=x_{n+1}=\left(x_{n+1}^{j}\right)_{j \in J}$. The current move $x \curvearrowright y$ is a change (resp. stay), if $y \neq x$ (resp. $y=x$ ). The current motivation to change of agent $j$ is $M_{e}^{j}(x, y)=U_{e}^{j}\left(A_{e}^{j}(x, y)\right)$, where $U_{e}^{j}\left(A_{e}^{j}\right)$ is the utility of his advantages to change $A_{e}^{j}=A_{e}^{j}(x, y)$. His current resistance to change is $R_{e}^{j}(x, y)=D_{e}^{j}\left(I_{e}^{j}(x, y)\right)$, where $D_{e}^{j}\left(I_{e}^{j}\right)$ is the disutility of his inconveniences to change $I^{j}=I^{j}(x, y)$. In the current period, each agent $j$ will find that such an organizational change is worthwhile if his current motivation to change is not lower than his current resistance to change, i.e., $M_{e}^{j}(x, y) \geq \lambda^{j} R_{e}^{j}(x, y)$ for all $j \in J$, where the higher is the current individual worthwhile to change ratio $\lambda^{j}=\lambda_{n+1}^{j}>0$, the more it is worthwhile to change for agent $j$. In this case, no agent will resist to change or block change. Let

$$
W_{e, \lambda}(x)=\left\{y \in X: M_{e}^{j}(x, y) \geq \lambda^{j} R_{e}^{j}(x, y), j \in J\right\}
$$

be the worthwhile to change set of the group at $x$. Our present paper considers a specific instance of this general model. To see how this is the case, please, see Sect. 4.2 just below. In this case, among other aspects, experience does not matter much and all worthwhile to change ratios are equal to a constant. Notice that experience have nothing to do with the vector e given in Sect. 3. Then, a worthwhile stay and change approach or avoidance group dynamic is defined by a succession of worthwhile stays and changes for the group, $x_{n+1} \in W_{e_{n}, \lambda_{n+1}}\left(x_{n}\right)$, for all $n \in \mathbb{N}$, where $\lambda_{n+1}=\left(\lambda_{n+1}^{j}\right)_{j \in J}$.

In this general (VR) context, a worthwhile stay and change group dynamic can end in a variational trap, before being able to reach a desired end for the group. The (VR) approach defines variational traps $x_{*} \in X$ for a group as, both,

(i) aspiration points, worthwhile to approach, $x_{n+1} \in W_{e_{n}, \lambda_{n+1}}\left(x_{n}\right), \forall n \in \mathbb{N}, x_{n} \rightarrow$ $x_{*}, n \rightarrow \infty$ and reach $x_{*} \in W_{e_{n}, \lambda_{n+1}}\left(x_{n}\right), \forall n \in \mathbb{N}$, from any or some successive position of the group and,

(ii) stationary traps, not worthwhile to leave to any member of the group, i.e., $W_{e_{*}, \lambda_{*}}\left(x_{*}\right)=\left\{x_{*}\right\}$, where $e_{*}$ and $\lambda_{*}$ are the current experiences and worthwhile to change ratio at $x_{*}$.

\subsection{A Benchmark Behavioral Context}

Let us show how our extension of EVP gives sufficient conditions for the existence of variational traps for a group which can meet desires or not, depending of the context. The present mathematical extension of EVP considers a specific but very important benchmark version of the previous general (VR) model of human behavior. It supposes that,

(i) experience does not matter much, i.e., $M_{e}^{j}(x, y)=U^{j}\left[A^{j}(x, y)\right]$ for all possible experience $e$ and each period $n+1$, 
(ii) motivation and resistance to change are identified to advantages and inconveniences to change, i.e., $U^{j}\left(A^{j}\right)=A^{j}$ and $D^{j}\left(I^{j}\right)=I^{j}$,

(iii) advantages to change are separable, i.e.,

$$
A^{j}(x, y)=g^{j}(y)-g^{j}(x)=f^{j}(x)-f^{j}(y), \forall j \in J,
$$

(iv) inconveniences to change are separable and nonnegative, i.e.,

$$
I^{j}(x, y)=C^{j}(x, y)-C^{j}(x, x) \geq 0, \forall j \in J,
$$

(v) worthwhile to change ratios are the same for each agent, and do not change from period to period, i.e., $\lambda_{n+1}^{j}=\lambda$ for all $j \in J$ and all $n \in \mathbb{N}$.

Let us explain a little more point (iii) and (iv). For much more explanations and examples, see [6]. Individual advantages to change $A^{j}(x, y)$ refer, each period, for each agent $j$, to the difference between his "to be increased" payoff $g^{j}(y)$ when all agents move and his to be increased "payoff $g^{j}(x)$ when all agents stay (repeat their last action). For example, $g^{j}(x)$ can be a profit. Let $\bar{g}^{j}=\sup \left\{g^{j}(z), z \in X\right\}<+\infty$ be the highest payoff agent $j$ can get within the group. Then, $f^{j}(y)=\bar{g}^{j}-g^{j}(y)$ defines his degree of unsatisfaction (a "to be decreased" payoff) and $A^{j}(x, y)=$ $g^{j}(y)-g^{j}(x)=f^{j}(x)-f^{j}(y)$. When they are nonnegative, inconveniences to change $I^{j}(x, y) \geq 0$ refer, each period, for each agent $j$, to the difference between his costs to be able to change $C^{j}(x, y) \geq 0$ and his costs to be able to stay $C^{j}(x, x) \geq 0$. The (VR) approach has shown, very carefully, how each individual inconveniences to change function $I^{j}(x, y)=C^{j}(x, y)-C^{j}(x, x) \geq 0$ can be modeled as a scalar pseudo-quasidistance, which is zero for a stay and is nonnegative for a change, with the possibility that $I^{j}(x, y)=0$ when $y \neq x$. Hence, if we want to model group dynamics, the space of actions or situations $X$ must be endowed with a cone pseudoquasimetric $q_{\Theta}(x, y)=I(x, y)=\left(I^{j}(x, y)\right)_{j \in J}=C(x, y)-C(x, x)$, which models vectors of inconveniences to change, where $C(x, y)=\left(C^{j}(x, y)\right)_{j \in J}$ and $C(x, x)=$ $\left(C^{j}(x, x)\right)_{j \in J}$ refer to vectorial costs to be able to change and to stay. The cone puts weights on the vector of motivations and resistances to change and helps to say when the vector of motivations to change is not lower than the vector of resistance to change with respect to some order relation.

Let $g(x)=\left(g^{j}(x)\right)_{j \in J}, f(x)=\left(f^{j}(x)\right)_{j \in J}$, be "to be increased" and "to be decreased" vectorial payoffs, and $A(x, y)=g(y)-g(x)=f(x)-f(y)$ be vectorial advantages to change. Then, it is easy to see that our extension of EVP to cone pseudoquasimetric spaces gives sufficient conditions for the existence of variational traps. More precisely, Theorem 3.1 shows that,

(i) $f\left(x_{0}\right)-f\left(x_{*}\right) \geq_{\Theta} \lambda q_{\Theta}\left(x_{0}, x_{*}\right)$ i.e., $A\left(x_{0}, x_{*}\right) \geq_{\Theta} \lambda I\left(x_{0}, x_{*}\right) \Longleftrightarrow x_{*} \in$ $S_{f, \lambda}\left(x_{0}\right)$. This means that it is worthwhile to move from $x_{0}$ to $x_{*}$. The proof of Theorem 3.1 shows more: $x_{*} \in S_{f, \lambda}\left(x_{n}\right)$ for all $n \in \mathbb{N}$, i.e., $x_{*}$ is an aspiration point,

(ii) $f\left(x_{*}\right)-f(x) \Varangle_{\Theta} \lambda q_{\Theta}\left(x_{*}, x\right)$ i.e., $A\left(x_{*}, x\right) \Varangle_{\Theta} \lambda I\left(x_{*}, x\right) \Longleftrightarrow x \notin S_{f, \lambda}\left(x_{*}\right)$ for all $x \in X \backslash \overline{\left\{x_{n}\right\}}$. Then, it is not worthwhile to leave $x_{*}$, except to move to $\overline{\left\{x_{n}\right\}}$ with a zero cost.

Then, $x_{*}$ is a variational trap. 


\section{Conclusions}

The cone pseudo-quasimetric versions of EVP obtained in this paper are motivated by a wide range of applications in group dynamics. They reduce to the corresponding results in [23] when the cone pseudo-quasimetric in question is $\left(X, \mathbb{R}, \mathbb{R}_{+}, q\right)$. Note that since the (scalar) pseudo-quasimetric versions of EVP were proved as characterizations of completeness of (scalar) pseudo-quasimetric spaces, a similar result could be verified in cone pseudo-quasimetric spaces. Note also that our results can give immediate extensions of fixed-point theorems in cone pseudo-quasimetric spaces.

Although we do not require that the ordering cone is normal or pointed, it is assumed to be solid. In future research, we would study the possibility of dropping this requirement since many versions of EVP in [19-21] were formulated for nonsolid ordering cones by using a variational approach.

Acknowledgments The contribution of the first author was completed while he visited Aix-Marseille University with a GREQAM-AMSE Grant. The authors are gratefully indebted to the anonymous referee for his/her helpful remarks, which allowed us to improve the original presentation.

\section{References}

1. Ekeland, I.: Sur les problémes variationnels. C. R. Acad. Sci. Paris 275, 1057-1059 (1972)

2. Lewin, K.: Frontiers in group dynamics: concept, method and reality in social science; social equilibria and social change. Hum. Relat. 1, 5-41 (1947)

3. Lewin, K.: Field Theory in Social Science. Harper and Row, New York (1951)

4. Soubeyran, A.: Variational rationality, a theory of individual stability and change: worthwhile and ambidextry behaviors. Preprint at GREQAM, Aix-Marseille University (2009)

5. Soubeyran, A.: Variational rationality and the "unsatisfied man": routines and the course pursuit between aspirations, capabilities and beliefs. Preprint at GREQAM, Aix-Marseille University (2010)

6. Soubeyran, A.: Variational rationality, part 1. Worthwhile stay and change approach-avoidance human dynamics ending in traps. Preprint at GREQAM-AMSE, Aix-Marseille University (2016)

7. Townsend, J.T., Busemeyer, J.R.: Approach-avoidance: return to dynamic decision behavior. In: Izawa, C. (ed.) Cognitive Processes the Tulane Flowerree Symposia on Cognition, p. 107. Lawrence Erlbaum Associates (2014)

8. Abdeljawad, T., Karapinar, E.: Quasicone metric spaces and generalizations of Caristi-Kirk's theorem. Fixed Point Theory Appl. 1, 574387 (2009). doi:10.1155/2009/574387

9. Lin, L.J., Wang, S.Y., Ansari, Q.H.: Critical point theorems and Ekeland type variational principle with applications. Fixed Point Theory Appl. 2011, 914624 (2011). doi:10.1155/2011/914624

10. Kazeem, E.F., Agyingi, C.A., Gaba, Y.U.: On pseudo-quasimetric type spaces. Chin. J. Math. 2014, 198685 (2014). doi:10.1155/2014/198685

11. Amini-Harandi, A., Fakhar, M.: Fixed point theory in cone metric spaces obtained via the scalarization method. Comput. Math. Appl. 59(11), 3529-3534 (2010)

12. Cho, S.H., Bae, J.S.: Variational principles on cone metric spaces. Int. J. Pure Appl. Math. 77(5), 709-718 (2012)

13. Du, W.S.: A note on cone metric fixed point theory and its equivalence. Nonlinear Anal. 72(5), 2259$2261(2010)$

14. Kadelburg, Z., Radenović, S., Rakočević, V.: A note on the equivalence of some metric and cone metric fixed point results. Appl. Math. Lett. 24(3), 370-374 (2011)

15. Janković, S., Kadelburg, Z., Radenović, S.: On cone metric spaces: a survey. Nonlinear Anal. 74(7), 2591-2601 (2011)

16. Shaddad, F., Noorani, M.S.M.: Fixed point results in quasi-cone metric spaces. Abstr. Appl. Anal. 2013:303626, 1-7 (2013). doi:10.1155/2013/303626 
17. Asadi, M., Rhoades, B., Soleimani, H.: Some notes on the paper "the equivalence of cone metric spaces and metric spaces”. Fixed Point Theory Appl. 2012:87, 1-4 (2012). doi:10.1186/1687-1812-2012-87

18. Feng, Y., Mao, W.: The equivalence of cone metric spaces and metric spaces. Fixed Point Theory 11(2), 259-264 (2010)

19. Bao, T.Q., Mordukhovich, B.S., Soubeyran, A.: Variational analysis in psychological modeling. J. Optim. Theory Appl. 164, 290-315 (2015)

20. Bao, T.Q., Mordukhovich, B.S., Soubeyran, A.: Fixed points and variational principles with applications to capability theory of wellbeing via variational rationality. Set-Valued Var. Anal. 23, 375-398 (2015)

21. Bao, T.Q., Mordukhovich, B.S., Soubeyran, A.: Minimal points, variational principles, and variable preferences in set optimization. J. Nonlinear Convex Anal. 16(8), 1511-1537 (2015)

22. Bao, T.Q., Théra, M.: On extended versions of Dancs-Hegedüs-Medvegyev fixed point theorem. Optimization (2015). doi:10.1080/02331934.2015.1113533

23. Bao, T.Q., Cobzaş, S., Soubeyran, A.: Variational principles, completeness and the existence of traps in behavioral sciences (2015) (Preprint at Optimization-Online), p. 25

24. Wilson, W.A.: On quasi-metric spaces. Am. J. Math. 3, 675-684 (1931)

25. Kelly, J.C.: Bitopological spaces. Proc. Lond. Math. Soc. 13, 71-89 (1963)

26. Reilly, I.L., Subrahmanyam, P.V., Vamanamurthy, M.K.: Cauchy sequences in quasi pseudometric spaces. Mon. Math. 93, 127-140 (1982)

27. Cobzaş, S.: Completeness in quasi metric spaces and Ekeland variational principle. Topol. Appl. 158, 1073-1084 (2011)

28. Karapinar, E., Romaguera, S.: On the weak form of Ekeland's variational principle in quasi-metric spaces. Topol. Appl. 184, 54-60 (2015)

29. Gert (Tammer), C., Weidner, P.: Nonconvex separation theorems and some applications in vector optimization. J. Optim. Theory Appl. 67, 297-320 (1990)

30. Göpfert, A., Riahi, H., Tammer, C., Zălinescu, C.: Variational Methods in Partially Ordered Spaces. Springer, New York (2003)

31. Khanh, P.Q.: On Caristi-Kirk's theorem and Ekeland's variational principle for pareto extrema. Bull. Pol. Acad. Sci. Math. 37, 33-39 (1989)

32. Németh, A.B.: Ekeland's variational principle in ordered abelian groups. Nonlinear Anal. Forum 6, 299-312 (2001)

33. Gutiérrez, C., Jiménez, B., Novo, V.: A set-valued Ekeland's variational principle in vector optimization. SIAM J. Control Optim. 47, 883-903 (2008)

34. Tammer, C., Zălinescu, C.: Vector variational principles for set-valued functions. Optimization 60, 839-857 (2011)

35. Qiu, J.H.: Set-valued quasimetrics and a general Ekeland's variational principle in vector optimization. SIAM J. Control Optim. 51, 1350-1371 (2013) 